\title{
Comment on Grmec et al.: A treatment protocol including vasopressin and hydroxyethyl starch solution is associated with increased rate of return of spontaneous circulation in blunt trauma patients with pulseless electrical activity
}

\author{
Dee Kotak \\ Received: 16 November 2008 / Accepted: 30 November 2008 / Published online: 23 January 2009 \\ (C) Springer-Verlag London Ltd 2009
}

\section{Dear Editor,}

The study by Grmec et al. is encouraging given the very high mortality of trauma victims who experience out-ofhospital pulseless electrical activity (PEA) arrest $[1,2]$. In the treatment group there were three interventions: vasopressin, hypertonic saline and hydroxyethyl starch (hypertonic hydroxyethyl starch in the study). However, caution is to be advocated in the use of hydroxyethyl starch (HES) as the preferred colloid in this clinical setting. The recently published VISEPT study (10\% $200 \mathrm{kDa} / 0.5 \mathrm{HES})$ and others (10\% $200 \mathrm{kDa} / 0.62 \mathrm{HES})$ have shown an association of HES with increased frequency of renal impairment in sepsis and also in renal transplantation [3-5]. A systematic review concluded that HES increases the risk of acute renal failure among patients with sepsis and may also reduce the probability of survival [6]. With the overwhelming renal insult from a haemorrhagic PEA arrest, the addition of the vasoconstrictive effects of vasopressin and an absence of solid evidence for benefit of HES, it would be better to use a colloid not associated with renal dysfunction. The newer starches are smaller, less substituted $(130 \mathrm{kDa} / 0.4)$ and may be safer than the ones used in previous studies but the uncertainty remains.

Fluid replacement is established in controlled haemorrhagic shock, but its use in uncontrolled haemorrhagic shock is still controversial. In traumatic PEA the primary

D. Kotak $(\bowtie)$

Intensive Care Medicine, Department of Anaesthesia,

King's College Hospital,

Denmark Hill,

London SE5 9RS, UK

e-mail: deekotak@yahoo.co.uk goal is to restore blood pressure and cardiac output to a level that can sustain life and prevent secondary cerebral injury. Given that haemorrhage is the most likely cause of the PEA, then judicious administration of fluid in combination with vasopressin is physiologically sound as the shift in blood away from subdiagphragmatic structures towards the heart and brain, mediated by the administration of vasopressin, may not be sufficient on its own to restore spontaneous circulation. In such an acute extreme pathophysiological situation it is more likely that the provision of intravascular volume is more important than the type of fluid. The debate over crystalloid vs colloid and colloid vs colloid continues and is now confounded by the increasing interest in the use of hypertonic saline in trauma.

An international, multicentre, randomised controlled trial to assess the effects of vasopressin vs saline placebo in prehospital traumatic haemorrhagic shock patients not responding to standard shock treatment will commence in January 2009 [Vasopressin in traumatic haemorrhagic shock (VITRIS.at) study] [7, 8]. The purpose of the trial is to assess the effect of vasopressin on hospital admission rate (primary study end point), as well as on haemodynamic variables, fluid resuscitation requirements and hospital discharge rate (secondary study end points), in pre-hospital, presumed traumatic haemorrhage shock patients with a systolic arterial blood pressure below $90 \mathrm{mmHg}$ after more than $10 \mathrm{~min}$ but less than $30 \mathrm{~min}$ of standard shock treatment by the emergency medical physician [intubation, crystalloid, colloid and hypertonic saline (up to $4 \mathrm{ml} / \mathrm{kg}$ ) fluid resuscitation, and catecholamines (ephedrine, phenylephrine, norepinephrine)]. The trial is scheduled to complete in April 2011 and the results may obviate the need for further studies of vasopressin in traumatic PEA. 


\section{References}

1. Grmec S, Strnad M, Čander D, Mally S (2008) A treatment protocol including vasopressin and hydroxyethyl starch solution is associated with increased rate of return of spontaneous circulation in blunt trauma patients with pulseless electrical activity. Int J Emerg Med 1:311-316. doi:10.1007/s12245-008-0073-8

2. Martin SK, Shatney CH, Sherck JP et al (2002) Blunt trauma patients with prehospital pulseless electrical activity (PEA): poor ending assured. J Trauma 53:876-880

3. Brunkhorst FM, Engel C, Bloos F et al (2008) Intensive insulin therapy and pentastarch resuscitation in severe sepsis. N Engl $\mathrm{J}$ Med 358:125-139
4. Cittanova ML, Leblanc I, Legendre C et al (1996) Effect of hydroxyethylstarch in brain-dead kidney donors on renal function in kidney-transplant recipients. Lancet 348:1620-1622

5. Schortgen F, Lacherade JC, Bruneel F et al (2001) Effects of hydroxyethylstarch and gelatin on renal function in severe sepsis: a multicentre randomised study. Lancet 357:911-916

6. Wiedermann CJ (2008) Systematic review of randomized clinical trials on the use of hydroxyethyl starch for fluid management is sepsis. BMC Emerg Med 8:1

7. Lienhart HG, Wenzel V, Braun $J$ et al (2007) Vasopressin for therapy of persistent traumatic hemorrhagic shock: the VITRIS.at study (in German). Anaesthesist 56:145-148

8. http://clinicaltrials.gov/ct2/show/NCT00379522 